\title{
LORCA Y SU FLAMENCO: UNA APROXIMACIÓN ETNO-PERFORMATIVA AL PROCESO DE CREACIÓN Y RECEPCIÓN DE UN ESPECTÁCULO
}

\author{
Francisco Javier Bethencourt Llobet \\ Universidad Complutense de Madrid
}

Fecha de recepción: 04/11/2020

Fecha de aceptación: 26/05/2021

\section{Resumen}

Federico García Lorca y su relación con el flamenco ha sido investigada infinidad de veces por flamencólogos, poetas, antropólogos, etnomusicólogos, sociólogos, etc. Sin embargo, no ha sido estudiada en profundidad desde un punto de vista de la producción musical. Teniendo como referentes los discos y espectáculos de Enrique Morente, Eva Yerbabuena, etc., jóvenes bailaoras, músicos y musicólogos de diversas procedencias coincidimos en un contexto cultural bastante alejado de Granada, el nordeste de Reino Unido, y creamos un espectáculo llamado Lorca y su Flamenco que giraría por Leeds, North Tyneside y York. Al igual que otras producciones flamencas multiculturales, esta fue concebida como un proceso colaborativo con ensayos realizados en un contexto, Newcastle upon Tyne, y puesta en escenas en otros. Tras un nuevo trabajo de campo realizado con los informantes-participantes de aquel proyecto, analizaremos en este artículo el proceso de creación y recepción que tuvo especialmente para el público británico.

Palabras clave: Lorca, interdisciplinaridad, performance, producción, tecnología.

\section{LORCA AND HIS FLAMENCO: AN ETHNO-PERFORMATIVE APPROACH TO THE PROCESS OF CREATION AND RECEPTION OF A PERFORMANCE}

\begin{abstract}
The relationship between Federico García Lorca and his flamenco has been researched on many occasions by poets, anthropologists, ethnomusicologists, sociologists, and specialists in flamenco, etc. However, it has not been researched in depth from a production point of view. Using albums and performances by Enrique Morente, Eva Yerbabuena, and others as a reference, young dancers, musicians, and musicologists from different backgrounds worked together in a cultural context to put together a performance called Lorca y su Flamenco, not in Granada, but in the Northeast of the United Kingdom, which toured through the cities of Leeds, North Tyneside, and York. Similar to other multicultural flamenco performances, it was conceived as a collaborative project, with rehearsals in Newcastle upon Tyne, which was then
\end{abstract}


Enclaves. Revista de Literatura, Música y Artes Escénicas, n. ${ }^{\circ}$ 1, 2021, pp. 117-124. e-ISSN 2792-7350

Francisco Javier Bethencourt Llobet, «Lorca y su flamenco: una aproximación

performed in other cities. Having carried out fieldwork with the participants of those events, in this article I will analyse the process of creation and the reception of the audience, in particular, the British audience.

Keywords: Lorca, Interdisciplinarity, Performance, Production, Technology.

\section{Sumario}

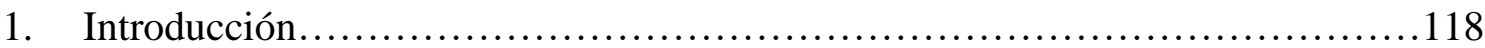

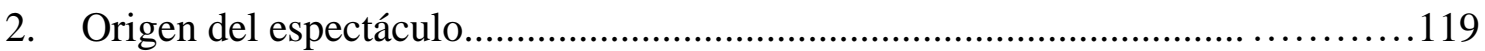

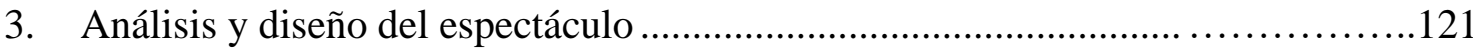

4. Reflexiones finales: ¿por qué es necesario seguir interpretando a Lorca? ............. 122

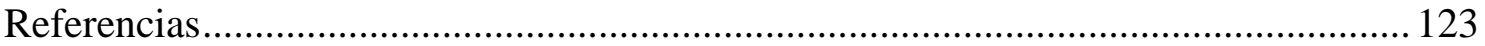

\section{Introducción}

Llueve a cántaros. El público sigue entrando en un pequeño teatro de York, está a punto de abrirse el telón cuando dos académicos, que hacen de poeta(s), se sientan detrás de una mesa. Vemos a la bailaora principal del espectáculo estirando, a un guitarrista calentando sus dedos, mientras el cajonero-percusionista le hace señas al técnico de sonido. Suena una flauta de lejos y las luces íntimas de dentro del escenario se encienden. Hay unos audiovisuales preparados como introducción al espectáculo. Nos encontrábamos en el teatro Upstage de York un 22 de mayo de 2011, junto a compañeras y compañeros de la Universidad de Newcastle, músicos profesionales y técnicos de luces y sonido, para presentar lo que sería el segundo concierto de Lorca y su Flamenco, que giraría por el norte de Inglaterra. Entre el público que va llenando la sala, nos encontramos a familiares, aficionados al flamenco de los colectivos de Leeds, Newcastle y York, así como aficionados a la poesía en general. La edad media del público es algo madura, cosa que no nos sorprende. Tras una breve introducción audiovisual que ayudaba a contextualizar a Federico García Lorca y el flamenco de su época, uno de los académicos recita un poema y la bailaora le responde con un lento movimiento de sus brazos. El taconeo estaría por llegar. Los músicos estaban colocados en el habitual semicírculo flamenco, aunque esta vez incluíamos una mesa para los «poetas». Los músicos se disponían a tocar entre micrófonos, monitores y cables que salían por todas partes. La mesa de monitores conectada a la mesa de mezclas se escondía detrás de unas cortinas. Poesía, música y tecnología se daban la mano en aquella aparentemente sencilla puesta en escena.

Como adelantábamos en las II Jornadas Internacionales de Investigación en Producción Musical, ${ }^{1}$ para que una puesta en escena como la que presentamos en York suceda se requiere de mucha preparación, esfuerzo y trabajo colectivo, además de un buen equipo técnico. Tras nuevas entrevistas realizadas con la bailaora y los músicos que participaron en aquella minigira, analizaremos cómo fue el proceso de creación del espectáculo, y sobre todo cuál fue la recepción del público británico. Tendremos en cuenta la implicación de una serie de

\footnotetext{
${ }^{1}$ En las II Jornadas Internacionales de Investigación en Producción Musical celebradas los días 26 y 27 de marzo de 2020, organizadas por Marco Juan de Dios y Jordi Roquer en la Facultad de Geografía e Historia de la Universidad Complutense de Madrid, aunque finalmente se realizaron online el 11, 12 y 13 de julio de 2020 por la COVID. En esta ponencia adelantábamos cómo productores como Alejandro Acosta preparaban con detalle sus performances, como la que vimos en el teatro Circo Price de Madrid.
} 
Enclaves. Revista de Literatura, Música y Artes Escénicas, n. ${ }^{\circ}$ 1, 2021, pp. 117-124. e-ISSN 2792-7350

Francisco Javier Bethencourt Llobet, «Lorca y su flamenco: una aproximación etno-performativa al proceso de creación y recepción de un espectáculo», https://dx.doi.org/10.12795/enclaves.2021.i01.07

académicos que contribuyeron notablemente al espectáculo. Aunque pueda parecer una aproximación meramente etnomusicológica, la perspectiva de los cultural performance studies y popular music studies son una referencia. Algunos compañeros musicólogos - Ian Biddle (2019), Kiko Mora (2008) o Marco Antonio Juan de Dios (2020) - que estudian la performance desde la producción musical asociada a cuestiones de identidad, género y queer theories han sido modelos a los que seguir en este proyecto.

\section{Origen del espectáculo}

En primer lugar, para entender el proyecto de Lorca y su flamenco es necesario comprender de dónde proviene la idea primigenia y la procedencia de sus miembros, ya que estamos ante un grupo multicultural de bailaoras, músicos, que provienen de España, Inglaterra y Venezuela, contextos culturales muy diferenciados que rápidamente nos conectan con conceptos de autenticidad, identidad y desplazamiento. Recuerdo que cuando vivía en Newcastle upon Tyne recibí una llamada de la bailaora Carla Soto, que en aquella época vivía en York. Impartía clases de flamenco y organizaba numerosos eventos junto a la Peña flamenca de Leeds. En la entrevista telemática que realicé a esta bailaora, que actualmente vive en Canadá, le preguntaba cómo se le había ocurrido la idea del espectáculo. Según sus propias palabras:

Yo siempre estuve enamorada de Lorca. Estudié letras. Tú me conoces y sabes que de toda la vida he bailado pero yo conocí a Lorca desde muy pequeñita a través de mi abuelo. Él siempre, después de su trabajo, me recitaba sus poemas, así que yo crecí con amor a él [Lorca]. Me llegaban sus palabras y sus sentimientos. (Entrevista personal)

Carla Soto, nacida en Kent un 21 de agosto de 1977, se crió en Venezuela y su formación es esencial para comprender el espectáculo y cómo adquirió su conocimiento sobre el poeta. La importancia de la familia, que hemos trabajado en músicos como Paco de Lucía, Gerardo Núñez y Enrique Heredia, Negri, se relaciona con una primera escena «glocal», utilizando el concepto de Robertson, donde hay una serie de elementos identitarios. Aparentemente, por haber nacido en un contexto cultural determinado parece que uno debe absorber una serie de códigos; sin embargo, por otro lado, absorbemos música y poesía que provienen de otros contextos culturales que hacemos nuestra, las asimilamos y luego vuelven a emerger a través de nuestros espectáculos. Esta idea sobre la adquisición del conocimiento la trabajamos en la tesis doctoral (Bethencourt, Rethinking Tradition...) donde analizamos lo esencial que es el conocimiento que adquirimos por el contexto cultural, la familia, pero también el que obtenemos al escuchar vinilos, CD o vídeos de otros géneros. Para la bailaora anglovenezolana:

Cuando yo entro a estudiar en la Universidad, una de mis razones es aprender bien sobre nuestra Literatura hispanoamericana [...] y a la par seguir bailando flamenco, mi pasión. Mi idea era, cuando me graduara, hacer un trabajo que relacionara la literatura y el baile. No lo tenía muy claro al principio, estaba buscando algo sobre poesía y flamenco que relacionara mis dos mundos, mis dos partes, mis dos caras. Un día, en casa de un amigo, buscando en su biblioteca descubro el Poema del Cante Jondo de Federico García Lorca y dije: « $i$ Wow, cante jondo, esto es flamenco!». Lo leo y me encuentro que hay mucha imaginería flamenca y trato de investigar. Recuerdo que en aquella época no había mucho Google [sic] y esos navegadores [...]. Una profesora me dijo que ese libro no tuvo buenas críticas, ya que era considerado un libro de juventud y que realmente Lorca se consagraría con el Romancero Gitano y con sus obras de teatro. A este conjunto de poemas no se les habían prestado demasiada atención y algunos 
Enclaves. Revista de Literatura, Música y Artes Escénicas, n. ${ }^{\circ}$ 1, 2021, pp. 117-124. e-ISSN 2792-7350

Francisco Javier Bethencourt Llobet, «Lorca y su flamenco: una aproximación etno-performativa al proceso de creación y recepción de un espectáculo», https://dx.doi.org/10.12795/enclaves.2021.i01.07

estudiosos no entendían qué quería aportar con esos poemas. Entonces empiezo a leerlo y siento que él quiere mostrar toda la imaginería de la muerte y el amor en el flamenco. Incluso incluye su famoso poema de la guitarra y otro sobre distintos palos [...]. Hablo con mi profesora de baile y creo que el libro tiene más sentido desde el punto de vista del baile que de la literatura. Por este motivo, decido que esta va a ser mi investigación, ya que es el vínculo entre los dos mundos [...]. (Entrevista personal)

Esta cita de la bailaora nos ayuda a adentrarnos en la construcción del universo que quiere mostrar la coproductora del espectáculo. El Poema del Cante Jondo ha sido escenificado innumerables veces; recuerdo una representación en la Chumbera con la Alhambra de fondo y con un cantaor recitando poesía, idea que incorporaríamos en nuestro espectáculo. Sin embargo, en Inglaterra recurrimos a dos académicos, Ian Biddle y Kiko Mora, que recitaban los poemas en castellano e inglés. Podemos analizar numerosos elementos identitarios, no solo andaluces o granadinos, de donde proviene el poeta, sino de las fuentes recogidas de la literatura oral y de la aportación de los académicos que formaron parte del proyecto. Kiko Mora, profesor de la Universidad de Alicante, que se encontraba en aquel momento de estancia en la Universidad de Newcastle, fue invitado a participar en el proyecto y entrevistado para el vídeo de contextualización del espectáculo. Kiko había realizado su tesis doctoral en EEUU sobre Las raíces del Duende: lo trágico y lo sublime en el cante jondo (2008), por lo que su perfil resultaba idóneo para formar parte del proyecto. Por otro lado, Ian Biddle, senior lecturer de la Universidad de Newcastle, también había investigado sobre flamenco y Lorca, llegando a publicar en Radical Musicology «Romance Cartógraphies», donde Lorca es analizado desde las queer theories. Sin embargo, hubo otros académicos que influyeron en la idea primigenia de este espectáculo. Según Carla Soto:

[...] Estaba en una Universidad de humanidades y nadie quería dirigir mi investigación porque decían que no sabían nada de baile. Me llamó una amiga de la Universidad científica y me dijo que había una electiva que se llama poesía y flamenco. Voy a la clase del profesor, Salvador Tenreiro, flamencólogo, que ahora vive en España, y nos hablaba de Machado que también se dedicó al flamenco [...]. Después de la clase el profesor me dice que efectivamente existe un vínculo entre Lorca y el flamenco, que fue parte importante de la organización del Concurso de Cante Jondo del año 1922 y que ayudó a promover el flamenco en una época en que este tenía mala connotación. Acepta a ser mi tutor y comenzamos a trabajar juntos. Me ayudó mucho. Al final presenté mi tesis y bailé acompañado por un guitarrista. El tema central de mi estudio era que para comprender el Poema del Cante Jondo tenías que comprender el mundo del flamenco [...]. (Entrevista personal)

Como podemos extraer de esta cita, Federico García Lorca y su obra son objeto de estudio en Universidades de Venezuela, EE. UU. e Inglaterra. Hay verdaderos especialistas en Lorca, como el hispanista Ian Gibson y jóvenes musicólogos como Marco Antonio de la Ossa. De haber publicado antes su Ángel, musa y duende: Federico García Lorca y la música (2014), nos habría sido una fuente de referencia, no solo para contextualizar el espectáculo sino por los arreglos transcritos de las canciones de Lorca. Carla Soto tampoco pudo contar estas referencias en Inglaterra, así que quiso contactar con un doctorando español. Aunque había vivido años en Granada, era un músico canario. Carla explica finalmente porqué nos contactó para coproducir el espectáculo:

Me quedé con ese libro, Poema del Cante Jondo, en la cabeza [...], así que cuando vivíamos en Inglaterra, recuerdo que tuvimos una oportunidad con James, que organizaba eventos en el 
Enclaves. Revista de Literatura, Música y Artes Escénicas, n. ${ }^{\circ}$ 1, 2021, pp. 117-124. e-ISSN 2792-7350

Francisco Javier Bethencourt Llobet, «Lorca y su flamenco: una aproximación etno-performativa al proceso de creación y recepción de un espectáculo», https://dx.doi.org/10.12795/enclaves.2021.i01.07

seven arts de Leeds y contigo, que estabas en Newcastle con el resto de españoles. Además, tú tocabas la guitarra, pero también investigabas y te interesabas por la historia del flamenco. Eres muy de teoría, no solamente de la parte práctica sino de la parte histórica. (Entrevista personal)

Como podemos leer en esta cita, la idea del espectáculo de Lorca y su flamenco está en la cabeza de la bailaora durante años y, sin embargo, necesitaba de un equipo para desarrollarlo y ponerlo en escena. Haciendo una reexposición, el espectáculo no surge como un encargo, sino que va más atrás en el tiempo. Cuando alguien te contacta para hacer una producción, hay ideas que incluso provienen de la infancia, como el caso de la bailaora. Al igual que nuestro toque no es algo que se invente para cada espectáculo, hay muchos años de estudio y conocimiento acumulado.

\section{Análisis y diseño del espectáculo}

Como jóvenes productores que no provenían de un ambiente cultural flamenco, queríamos incorporar elementos de flamenco «tradicional», pero intentábamos que no fuera la mímesis de los típicos tópicos y que incorporara elementos de nuestras identidades múltiples, así como elementos contemporáneos en el baile, toque y la escenografía. Sin embargo, teníamos los poemas y la estructura de Poema del Cante Jondo. Muchos de los poemas nos daban sus propios códigos y palos en los que «debíamos» interpretarlos. Por ejemplo, después de la presentación del vídeo introductorio en inglés, la guitarra comenzaba a sonar por tientos (palo binario) y a continuación se recitaba «El poema de la guitarra» (García Lorca 131), que, a pesar de encontrarse arropado por poemas sobre la seguiriya, usaríamos este palo para el baile que vendría a posteriori. Antes se recitaría el poema «Silencio» donde el resto de los músicos entrarían a escena. ${ }^{2}$

El poema del «Paso de la Seguiriya» es el momento donde la bailaora entra en el escenario. Al igual que la luz estaba centrada en la guitarra flamenca y el resto del escenario permanecía oscuro, ahora el baile se lleva todo el protagonismo, sobre todo estando en Inglaterra. Kiko Mora se encuentra recitando dicho poema. ${ }^{3}$ La seguiriya, con su compás a cinco golpes como lo marcan las bailaoras, se trata de un compás de amalgama de 12 tiempos ( 2 tiempos del $3 / 4$, un 3/4 y el último tiempo del 3/4). Este compás de amalgama lo hicimos como se suele tocar por medio, utilizando los acordes de Sib/Re, Do7/9, Sib y LaM (b9), para los flamencos la cadencia (IV - III - II - I). Los músicos británicos que colaboraban en el proyecto pensaban que estábamos tocando en Re menor y lo veían como un IV - V7 - IV - III (mayorizado), como lo conceptualiza Lola Fernández en su libro Teoría Musical del Flamenco (2004). Otros autores especialistas en flamenco prefieren hablar de cadencia andaluza, modo flamenco, Armonía modal, modo de Mi y flamenco, como titula su tesis doctoral Salvador Valenzuela (2016). Javier Suárez Pajares, en su defensa de cátedra, nos mencionaba que Manuel de Falla anotaba en sus partituras «como lo ven los flamencos, como lo ven los clásicos». ${ }^{4}$

\footnotetext{
${ }^{2}$ Paco comes on the stage and starts playing (tientos) while he is playing in the distance "La Guitarra» poem (recited by Kiko \& Ian) (2 min.). The Poem of «La Guitarra» in Spanish in the back (2 min.) [...]. «Silencio» poem (recited). After the poem finish all musicians come to the stage. Las notas a pie de página incluidas en inglés provienen del track list (lista de canciones) original del espectáculo.

${ }^{3}$ Poema «Paso de la Seguiriya» poem (recited by Ian first). Carla comes to the stage and dance the poem in Spanish when Kiko (2 min.) b (Seguiriya) dance by Carla. All musicians play (5 min.).

${ }^{4}$ Tribunal de Cátedra de Javier Suarez Pajares en la Universidad Complutense de Madrid. Más información sobre la relación entre Manuel de Falla y Federico García Lorca en Torres Clemente (2009).
} 
Enclaves. Revista de Literatura, Música y Artes Escénicas, n. ${ }^{\circ}$ 1, 2021, pp. 117-124. e-ISSN 2792-7350

Francisco Javier Bethencourt Llobet, «Lorca y su flamenco: una aproximación etno-performativa al proceso de creación y recepción de un espectáculo», https://dx.doi.org/10.12795/enclaves.2021.i01.07

Tras proyectar la traducción del poema «Pueblo» ${ }^{5}$ en la pantalla, hicimos «Cueva», ${ }^{6}$ que comenzaba por tarantos, y lo rematábamos por tangos de Granada. El poema «Cueva» nos recordaba a las zambras del Sacromonte. Quizá fuera un cliché, pero recuerdo el revuelo con los tangos (palo binario que solemos transcribir en $4 / 4$ o en $8 / 4$ incluyendo dos ciclos), que resulta más accesible al público británico.

Después de recitar el poema «Memento», ${ }^{7}$ hicimos las viñetas flamencas «El café Cantante», ${ }^{8}$ donde reinterpretábamos «Las coquinas» de la Niña de los Peines, con agilidad que cerraba la primera parte del espectáculo.

\section{- INTERMISSION-}

Después de un breve descanso, unos 15 minutos, empezamos la segunda parte del espectáculo con una malagueña que remataríamos por fandangos. El poema de «La Malagueña» ${ }^{9}$ fue recitado con su respectiva traducción para el público británico. La bailaora entraba nuevamente en escena con «La soleá», ${ }^{10}$ esta vez con bata de cola. La soleá, que es considerada «la madre del flamenco» para algunos aficionados, con sus doce tiempos estirados y flexibilizados por el cante y la guitarra, tomó rigidez en la escobilla y subida para volver al tempo lento que nos ayudaba a mostrar el ethos de la pérdida.

Lo que quedaba de espectáculo bajamos el nivel jondo, lo que realmente conmueve al público británico. En los espectáculos flamencos suelen combinar con otra serie de palos o melodías que forman parte de la memoria colectiva, que han hecho suyas compositores como Albéniz con «La Tarara». ${ }^{11}$ De la Leyenda del Tiempo recuerdo que hicimos «Los Jaleos», ${ }^{12}$ no por tientos como Enrique Morente, sino por bulerías. Finalizamos el espectáculo con «Verde que te quiero verde $»^{13}$ por rumbas al estilo de Manzanita. De bises recuerdo que metimos los cuatro muleros por bulerías, con sus pataítas finales por fiestas, e incluso llegamos a hacer «La Aurora de Nueva York», recordando al maestro granaíno y al guitarrista cordobés Vicente Amigo. ${ }^{14}$

\section{Reflexiones finales: ¿por qué es necesario seguir interpretando a Lorca?}

Muchas de las puestas en escena de flamenco están llenas de misticismo, relacionadas con una doble lectura del concepto de autenticidad (tradición y modernidad). Por ejemplo, en la Leyenda del Tiempo (1979), producida por Ricardo Pachón, en donde Camarón de la Isla cantaba a Lorca, se entiende perfectamente esa doble lectura del concepto de autenticidad

\footnotetext{
${ }^{4}$ «Pueblo» poem projected on the screen. Solo music and Cante (5 min.). It would be nice if singer could sing Lorca's poem.

${ }^{6}$ «Cueva» poem projected on the screen. Tarantos - tangos de Granada music and dance (7 min).

${ }^{7}$ «Memento» poem projected on the screen $(5 \mathrm{~min})$.

${ }^{8}$ From Viñetas Flamencas «El Café Cantante» poem projected on the screen. (Alegrías) «Coquinas» de la Niña de los Peines dance $(7 \mathrm{~min})$.

${ }^{9}$ Image of Andalucía. «Malagueña» poem projected on the screen. (Malagueña) intro and finish with fandangos (5 min.).

${ }^{10}$ «Soleá» poem ( $2 \mathrm{~min}$ ). Carla comes to the stage (Soleá) dance with bata de cola $(7 \mathrm{~min})$.

${ }_{11}$ «Amparo» poem on CD (1 min.). Solo guitar/or singing $(4 \mathrm{~min})$. «La Tarara».

${ }^{12}$ Jaleos «La Leyenda del Tiempo» music and dance $(4 \mathrm{~min})$.

${ }^{13}$ «Verde» por rumbas song and dance with castanets $(4 \mathrm{~min})$. Solo music and feedback with the audience. Carla dances at the end (5 $\mathrm{min})$.

${ }^{14}$ En aquel entonces, investigaba la figura de Vicente Amigo para mi tesis doctoral (Bethencourt, Rethinking Tradition...).
} 
Enclaves. Revista de Literatura, Música y Artes Escénicas, n. ${ }^{\circ}$ 1, 2021, pp. 117-124. e-ISSN 2792-7350

Francisco Javier Bethencourt Llobet, «Lorca y su flamenco: una aproximación etno-performativa al proceso de creación y recepción de un espectáculo», https://dx.doi.org/10.12795/enclaves.2021.i01.07

(Bethencourt, Rethinking Tradition...). José Fernández (alias Tomatito) esperaba que su primera grabación con Camarón fuera hacia la «tradición», un disco de cante con acompañamiento de guitarra. Como decía Tomatito: «Qué es lo que mi gente va a decir que están esperando verme grabar junto al maestro», y Camarón le respondía «No te preocupes, que cuando yo lo cante, sonará flamenco». En nuestro espectáculo sobre Lorca, la «autenticidad» no solo se la daba la cantaora, Lina León, sino los distintos miembros, Kiko Mora, Ian Biddle, Jeremy Bradfield con su toque contemporáneo de cajón, llevando a Lorca y sus poemas a nuevas aproximaciones al flamenco y el mestizaje. La riqueza del espectáculo Lorca y sus flamenco(s) era la múltiple identidad de sus protagonistas, en esa mezcla que ha sido conceptualizada por Gerard Steingress como «hibridación transcultural», donde cada músico aporta conforme a su cultura.

Quizá no respetamos el orden de los poemas, pero lo hicimos para que fluyera el espectáculo. Cuando nos preguntamos: «Y si hiciéramos el espectáculo de Lorca y su flamenco de nuevo, ¿qué haríamos distinto?». La repuesta de ambos fue: «La haríamos más social», y relacionada con lo que está sucediendo en la actualidad; por ejemplo, con el movimiento \#blackmatters, ya que el arte y la poesía, como la música, reflejan el momento social e histórico que vivimos. El flamenco también es una construcción social e histórica que está en constante evolución, absorbiendo todo aquello que la enriquece sin perder como referencia las raíces y los palos. A la respuesta de por qué es importante seguir interpretando a Lorca: mientras siga habiendo casos de violencia de género, discriminación racial o por cuestiones de tendencia sexual - a algún compañero lo han agredido por decir que es gay - creo que merece la pena seguir reinterpretándolo; no solo por la belleza y la universalidad de su obra sino por como murió. A Lorca no solo lo mataron por una cuestión política. Para el público británico, quizás Lorca no era parte de su identidad, pero sí lo eran los sentimientos allí presentados. Carla agradecía a los músicos, familia, espectadores y daba las gracias a su abuelo y a Lorca por ser los culpables del aquel tinglado.

\section{Referencias}

Bethencourt Llobet, Francisco. Rethinking Tradition: Towards an Ethnomusicology of Contemporary Flamenco Guitar. Tesis doctoral. Newcastle: Newcastle University Press, 2011. https://theses.ncl.ac.uk/jspui/handle/10443/1305. Web 3 Nov. 2020.

Bethencourt Llobet, Francisco y Eduardo Murillo Saborido. "El legado de Paco de Lucía: la transmisión del conocimiento en la guitarra flamenca contemporánea". Roseta, n. ${ }^{\circ}$ 14, 2019, pp. 82-103.

Biddle, Ian. "Romance Cartographies: Flamenco Articulations of Queer Spaces in Urban Andalusia”. Radical Musicology, vol. 7, 2019. Web 15 Oct. 2020.

Castro Buendía, Guillermo. "Del canto de columpio andaluz a La leyenda del tiempo de Camarón de la Isla". Sinfonía Virtual, n. ${ }^{\circ}$ 22, 2012. Web 30 Oct. 2020.

Chichilla, Gema. La influencia de Federico García Lorca en el flamenco. TFG. Madrid: Universidad Complutense de Madrid, 2020.

Cruces Roldán, Cristina. La Niña de los Peines: el mundo flamenco de Pastora Pavón. Sevilla: Almuzara, 2009.

De la Osa Martínez, Marco Antonio. Ángel, musa y duende: Federico García Lorca y la música. Madrid: Editorial Alpuerto, 2014.

Elliott, Richard. Fado and The Place of Longing: Loss, Memory and the City. Surrey: Ahsgate, 
Enclaves. Revista de Literatura, Música y Artes Escénicas, n.o 1, 2021, pp. 117-124. e-ISSN 2792-7350

Francisco Javier Bethencourt Llobet, «Lorca y su flamenco: una aproximación etno-performativa al proceso de creación y recepción de un espectáculo», https://dx.doi.org/10.12795/enclaves.2021.i01.07

2010.

Fernández, Lola. Teoría Musical del Flamenco. Madrid: Acordes Concert, 2004.

Mora, Francisco. Las raíces del Duende: lo trágico y lo sublime en el cante jondo. Tesis doctoral. Ohio State University, 2008.

Núñez, Faustino y José Manuel Gamboa. Camarón: Vida y Obra. Madrid: SGAE, 2003.

Soto, Carla. Entrevista personal (30 Sep. 2020).

Steingress, Gerhard. "La hibridación transcultural como clave de la formación del nuevo flamenco". Trans, n. ${ }^{\circ}$ 6, 2005, pp. 119-152. Web 20 Oct. 2020.

Torres Clemente, Elena. Manuel de Falla. Málaga: Editorial Arguval, 2009.

Valenzuela Lavado, Salvador. Armonía modal, modo de Mi y flamenco: aproximación al «Modo de Mi armónico» como sistema de tradición hispana. Tesis doctoral. Granada: Universidad de Granada, 2016. 